After 6 months of evolution all symptoms were resolved, with persisting hematuria in cases of nephropathy.

Conclusions HSP is usually a self-limiting disease. The extrarenal symptoms usually resolve quickly, without complications, and long-term prognosis depends mainly on the severity of renal involvement.

\section{PO-0356 HOW FREQUENT GET TUBERCULOSIS A CF PATIENT}

I Ciuca, L Pop. Pediatric II Department, "Victor Babes" University of Medicine and Pharmacy, Timisoara, Romania

\subsection{6/archdischild-2014-307384.1004}

Background Cystic fibrosis patients are predisposed to pulmonary infections. Conditions associated with $\mathrm{CF}$, like underweight, diabetes mellitus (CFRD), liver disease (CFLD) are favouring factors for tuberculosis (TB). The hypothesis of a potential comorbidity of TB in CF children occurred. The aim of the paper was to evaluate the prevalence of $\mathrm{TB}$ in children with complicated CF.

Methods Thirty-two patients (pts) with typical CF, associating complication like CFLD(27 pts), diabetes (3 pts) and 2 with both complication were considered for a prospective five years study. Biannual bacteriologic exam (TB specific also) were included, in addition to clinical examination and annual CT, to the patients evaluation.

Results Tuberculosis occurred in 6.25\% (2 patients), both F508 del homozygous, with CFLD and poor nutritional status; one patient had also CFRD. His evolution was unfavourable; he developed portal hypertension and died from respiratory failure. The other patient was diagnosed with active TB, Pseudomonas positive and poor nutritional status, but good evolution after treatment. The rest of CFLD patients had a stationary evolution, except 4 of them $(15.38 \%)$ developed diabetes. Tuberculin skin test was positive in 4 patients (12.5\%), 3 of them received TB vaccine. Despite the mandatory vaccination for TB in our country, only $84 \%$ pts were vaccinated. $18.75 \%$ of patients (6 pts) were considered and treated as TB cases, without bacteriological confirmation, before being diagnosed with CF.

Conclusion Although TB is a frequent condition in our area, the prevalence among CF children is not as high as expected. It is possible that other factors, unknown so far, are implicated.

\section{P0-0357 PRENATAL EXPOSURE TO ENDOCRINE DISRUPTING CHEMICALS (EDCS) IN RELATION TO THYROID HORMONE LEVELS IN INFANTS}

${ }^{1} \mathrm{M}$ de Cock, ${ }^{2} \mathrm{MR}$ de Boer, ${ }^{3} \mathrm{M}$ Lamoree, ${ }^{3} \mathrm{~J}$ Legler, ${ }^{1} \mathrm{M}$ van de Bor. ${ }^{1}$ Health and Life Sciences, VU University, Amsterdam, Netherlands; 'Health Sciences, VU University, Amsterdam, Netherlands; ${ }^{3}$ Institute for Environmental Studies, VU University, Amsterdam, Netherlands

\subsection{6/archdischild-2014-307384.1005}

Background The presence of chemicals in the environment is ubiquitous. EDCs in particular may disrupt thyroid hormones, which in early life are essential for brain development. As observational studies regarding this topic are still limited, the objective of the current study was to assess the association between prenatal exposure to various EDCs and thyroxine (T4) levels in newborns in a mother-child cohort in the Netherlands.
Methods Exposure to amongst others dichlorodiphenyldichloroethylene (DDE), perfluorooctanesulfonic acid (PFOS), and perfluorooctanoic acid (PFOA) was determined in cord plasma or breast milk, and information on T4 levels in heel prick blood spots was obtained through the Dutch neonatal screening programme. Linear regression models were composed for each compound and T4. Models were stratified for gender and adjusted for a priori defined covariates.

Results Mean T4 level was $86.9 \mathrm{nmol} / \mathrm{L}(\mathrm{n}=83)$. Girls in Q4 of DDE and PFOA exposure showed an increased T4 level compared to Q1, a difference which remained significant in the fully adjusted model (DDE $\geq 110.01 \mathrm{ng} / \mathrm{L},+22.2 \mathrm{nmol} / \mathrm{L}, 95 \% \mathrm{CI}$ $1.37,43.04 ;$ PFOA $\geq 1201 \mathrm{ng} / \mathrm{L},+30.7 \mathrm{nmol} / \mathrm{L}, 95 \%$ CI 13.38 , 48.05). In boys a lower T4 level was seen in Q2 for both PFOS and PFOA, however after adjusting the models these associations were attenuated.

Conclusion DDE, PFOS and PFOA were associated with T4 in a sex-specific manner. As the study population was relatively small, results should be considered as exploratory. More research is warranted, as studies on the role of exposure to environmental contaminants in thyroid hormone homeostasis are still limited.

\section{PO-0358 CONCOMITANT CEFTRIAXONE-INDUCED NEPHROLITHIASIS AND BILIARY PSEUDOLITHIASIS IN A SMALL TODDLER}

${ }^{1} \mathrm{~S}$ Diaconescu, ${ }^{1} \mathrm{~A}$ Baltag, ${ }^{2} \mathrm{C}$ Olaru, ${ }^{1} \mathrm{~N}$ Gimiga, ${ }^{1} \mathrm{M}$ Burlea, ${ }^{1} \mathrm{CA}$ Olaru, $1 \mathrm{I}$ Miron. "Pediatrics, "Gr. T. Popa" University of Medicine and Pharmacy, Jassy, Romania; ${ }^{2}$ Pediatrics, "St. Mary" Children's Emergency Hospital, Jassy, Romania

\subsection{6/archdischild-2014-307384.1006}

Introduction Ceftriaxone is a frequent choice in Romanian hospitals for empiric antimicrobial therapy because of its broad spectrum, long half-life, safety and tolerability. However, some relatively rare side effects as renal lithiasis and biliary pseudolithiasis may occur.

Case report A 5 month male toddler was admitted into our unit with extreme agitation and mild respiratory symptoms. The child had interstitial pneumonia and received ceftriaxone intravenously for 7 days in a county hospital. Clinical examination showed productive cough, fine crackles, mild systolic murmur and small crystals in the in diaper. Ultrasound examination of the kidneys revealed the presence of sediment in the bladder and a hyperechoic image of $4 \mathrm{~mm}$ in the gallbladder. Complete blood count, urea, creatinine, creatinine clearance, serum glucose, calcium (total and ionised), serum phosphorus, bilirubinemia, alkaline phosphatase were within normal limits. Urinalysis showed calcium carbonate deposit and increased calcium/creatinine ratio. Chest X-Ray revealed discrete bilateral interstitial infiltrate. The echocardiography found permeable foramen ovale and moderate left ventricular diastolic dysfunction. Treatment consist in suspending ceftriaxone administration, antispasmodics, hydrochlorothiazide $6,25 \mathrm{mg} /$ daily and adequate hydration. The child was dismissed after 3 days in good general condition, disappearance of bladder sediment but persistant gallbladder image that disappeared within 3 weeks.

Conclusions This is a particular case of a very young patient with renal and vesicular pseudolithiasis induced by ceftriaxone; the negative family history of kidney stones, a normal intake of vitamin D3 and normal liver and renal function excluded any other cause of lithiasis except the antibiotic itself. 